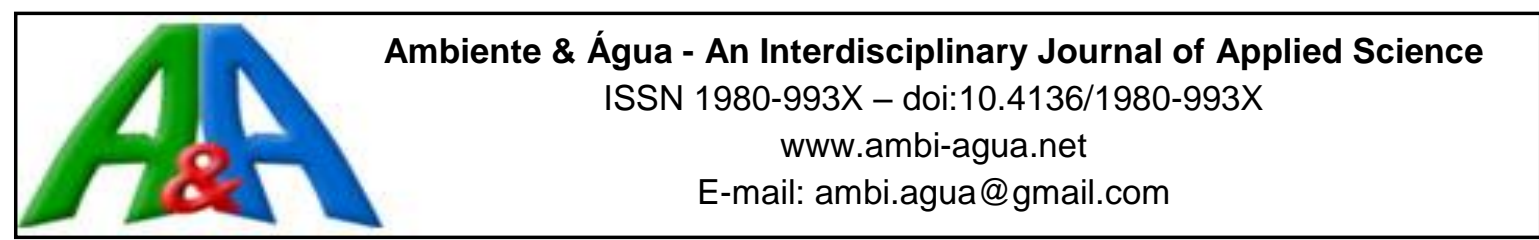

\title{
Determinação de hormônios estrogênicos em esgoto bruto e efluente de uma estação descentralizada de tratamento por lodos ativados
}

\author{
ARTICLES doi:10.4136/ambi-agua.2059
}

Received: 05 Dec. 2016; Accepted: 19 Dec. 2017

\begin{abstract}
Rossana Borges Teixeira*; Carolina Alves Marques; Natália Rodrigues de Carvalho; Luiz Eduardo Thans Gomes; Flávio Teixeira da Silva; Teresa Cristina Brazil de Paiva

\author{
Escola de Engenharia de Lorena (EEL-USP), Lorena, SP, Brasil \\ Departamento de Biotecnologia. E-mail: rossanabt@hotmail.com, carolina.4545@hotmail.com, \\ nataliadecarvalho@gmail.com, luizeduardo.gomes@hotmail.com, flavio@debiq.eel.usp.br, \\ teresa@debiq.eel.usp.br \\ *Autor correspondente
}

\section{RESUMO}

Hormônios estrogênicos, provenientes dos esgotos, atingem os corpos hídricos e podem causar perturbações aos organismos aquáticos. Os tratamentos secundários das estações de tratamento de esgoto (ETE) possibilitam alguma remoção destes compostos, mesmo em ETEs descentralizadas, apesar de ainda haver poucos estudos sobre elas. Em sistemas por lodos ativados, a eficiência tem sido relacionada ao tempo de detenção hidráulica (TDH), à idade de lodo, à desnitrificação biológica e à carga de alimentação. O objetivo deste trabalho foi quantificar os hormônios estrona (E1), 17- $\beta$-estradiol (E2), estriol (E3) e 17- $\alpha$-etinilestradiol (EE2) no esgoto e no efluente de uma ETE descentralizada por lodos ativados, além de caracterizar a ETE do Campus Universitário da EEL-USP quanto à matéria orgânica recebida, ao tempo de detenção hidráulica e a remoção de nitrogênio. Cromatografia líquida com detecção UV foi utilizada na determinação dos hormônios. Os resultados mostraram concentrações dos hormônios superiores às encontradas na literatura, em ambos: esgoto $(5,158$ $\pm 2,747 ; 7,434 \pm 4,356 ; 5,200 \pm 3,331$ e 5,638 $\pm 4,312 \mu \mathrm{g} \mathrm{L}^{-1}$ de E1, E2, E3 e EE2) e efluente $(5,062 \pm 3,366 ; 4,191 \pm 3,527 ; 7,743 \pm 3,951,2,550 \pm 2,162$ de E1, E2, E3 e EE2). No esgoto, as altas concentrações podem ser relacionadas à maior predominância de urina, visto os altos níveis de nitrogênio detectados, à menor geração de esgoto e a amostragem em período seco causando reduzida diluição dos hormônios. Remoção insuficiente dos hormônios devido a menor TDH (2h05) e irregularidade na desnitrificação (-54 a 61\%) pode ser o motivo das altas concentrações de estrógenos no efluente. Ademais, a desconjugação dos hormônios pode ter ocorrido durante o tratamento.

Palavras-chave: cromatografia líquida com deteç̧ão UV, esgoto de Campus universitário, estrógenos, ETE batelada, remoção de hormônios estrogênicos.

\section{Determination of estrogenic hormones in sewage and effluent of a decentralized sewage treatment plant by activated sludge}

\section{ABSTRACT}

Estrogenic hormones from sewers reach water bodies and can disrupt aquatic organisms. Secondary treatment of sewage treatment plants (STP) can remove some of those hormones, 
even in decentralized STPs, although there are few studies regarding this. In activated sludge systems, efficient removal has been attributed to hydraulic retention time (HRT), sludge age, biological denitrification and organic load. This study sought to quantify the hormones estrone (E1), 17 $\beta$-estradiol (E2), estriol (E3) and 17 $\alpha$-ethynylestradiol (EE2) in the sewage and effluent of a decentralized STP with activated sludge, and also to characterize this college campus STP regarding received organic matter, hydraulic retention time and nitrogen removal. Hormone levels were determined by liquid chromatography with UV detection. The results showed hormone concentrations far superior to those found in the literature in both the sewage (5.158 $\pm 2.747 ; 7.434 \pm 4.356 ; 5.200 \pm 3.331$ e $5.638 \pm 4.312 \mu \mathrm{g} \mathrm{L}^{-1}$ of E1, E2, E3 and EE2) and the effluent $(5.062 \pm 3.366 ; 4.191 \pm 3.527 ; 7.743 \pm 3.951,2.550 \pm 2.162$ of E1, E2, E3 and EE2). In the sewage, the high concentration could be related to a large predominance of urine, given the high level of nitrogen detected, or to low generation of sewage and to sample collection in dry periods causing decreased dilution of hormones. Insufficient removal of estrogens due to short HRT (2h05) and irregular denitrification (-54 to 61\%) can be the reason for high concentrations of estrogens found in the effluent. Furthermore, deconjugation of hormones may have occurred during treatment.

Keywords: batch WWTP, estrogens, liquid chromatography with UV detection, removal of estrogenic hormones, wastewater of College Campus.

\section{INTRODUÇÃO}

Os hormônios estrogênicos, inclusive os naturais, têm sido apontados como os responsáveis por grande parte das perturbações endócrinas em organismos aquáticos identificadas nos últimos anos. A relevância dos hormônios estrogênicos na perturbação endócrina se deve: à alta afinidade dos estrógenos aos receptores presentes nos organismos de outras espécies permitindo a ação, mesmo em baixíssimas concentrações (ng L $\mathrm{L}^{-1}$ ); à ampla excreção destes hormônios por seres humanos e por outros animais nas fezes e urina; e ao posterior despejo dos esgotos, tratados ou não, nos ecossistemas aquáticos (Carballa et al., 2004; Fonseca et al., 2013).

Diversos estudos realizados ao redor do mundo demonstraram que os esgotos municipais têm apresentado concentrações dos hormônios estrogênicos na faixa de ng $\mathrm{L}^{-1}$ e detecção dos hormônios pesquisados em quase todas as amostras. Já os hormônios artificiais têm sido determinados menos frequentemente, visto que a sua presença depende da prescrição e regulamentação para uso (Carballa et al., 2004; Janex-Habibi et al., 2009; Stanford e Weinberg, 2010; Fonseca et al., 2013; Ferreira, 2013).

Diferente dos esgotos domésticos, os efluentes de estações de tratamento de esgoto (ETE) apresentam menor concentração e menor frequência de detecção dos hormônios estrogênicos, inclusive os naturais. Estas reduções foram atribuídas, principalmente, à remoção destes compostos pelos tratamentos secundários adotados nas ETEs. A remoção dos hormônios engloba os processos de biotransformação conduzido pelos micro-organismos e a sorção dos compostos ao lodo, assim como ocorre com os constituintes majoritários dos esgotos (Carballa et al., 2004; Servos et al., 2005; Janex-Habibi et al., 2009; Stanford e Weinberg, 2010; Fonseca et al., 2013).

Estima-se que o sistema de lodos ativados seja o tratamento biológico mais utilizado no mundo e com isto, trabalhos sob a remoção de hormônios estrogênicos vêm sendo investigados neste tipo de tratamento (D'Ascenzo et al., 2003). Diferentes eficiências de remoção têm sido obtidas e os melhores resultados foram atribuídos a maior tempo de detenção hidráulica (TDH), prolongadas idade de lodo, baixa carga de alimentação e melhor remoção biológica de nutrientes, principalmente, o nitrogênio (Johnson et al., 2005; Ferreira, 2013). 
As ETEs descentralizadas, atendendo pequenas comunidades ou negócios, podem ser uma alternativa para a redução da poluição hídrica, porém podem apresentar riscos à qualidade da água se não forem efetivamente monitoradas. Nos Estados Unidos (USA), a participação das ETEs descentralizadas no tratamento de esgotos foi estimada em 25\% (Stanford e Weinberg, 2010). Apesar da relevância deste modelo de tratamento de esgotos, grande parte das publicações a respeito da presença e remoção de hormônios estrogênicos foi realizada em ETEs centralizadas. Uma exceção é o trabalho de Stanford e Weinberg (2010). Estes quantificaram e avaliaram a remoção dos hormônios estrogênicos em tratamentos descentralizados comumente utilizados nos USA: tanques sépticos, alagados aeróbios e anaeróbios e filtros de areia. Mesmo nestes tratamentos de operação mais simplificada, foram obtidas remoções dos hormônios superiores a $80 \%$.

O objetivo deste trabalho foi quantificar os hormônios estrogênicos naturais, E1, E2 e E3, e o hormônio artificial EE2, no esgoto e no efluente da ETE descentralizada com operação em batelada que atende a Escola de Engenharia de Lorena (EEL-USP), além de caracterizar a ETE quanto ao tempo de detenção hidráulica (TDH), carga orgânica de alimentação e desempenho na remoção de nitrogênio, visto o impacto destes fatores na remoção dos hormônios estrogênicos.

\section{MATERIAL E MÉTODOS}

\subsection{Local de estudo}

A ETE da EEL-USP utiliza lodos ativados em bateladas sequencias (SBR) para tratar todo o esgoto gerado no Campus I, em média $85 \mathrm{~m}^{3}$ por dia. O esgoto é proveniente de laboratórios, sanitários, vestiários, da limpeza de edifícios e da lavagem de utensílios do serviço de alimentação. Não há moradias no Campus e nem há preparo dos alimentos servidos no Restaurante Universitário.

A ETE em estudo foi, primeiramente, projetada para operar de forma contínua, porém o aumento na geração de esgoto, consequência do aumento do número de alunos, fez com que o regime de operação em bateladas fosse adotado. O modo SBR permitiu utilizar o decantador secundário também como tanque de reação/aeração, aumentando a capacidade da ETE. A ETE possui duas particularidades: (i) não possui tratamento preliminar como outras ETEs sanitárias instaladas na região, que possuem ao menos caixa para a remoção de areia e possuem grades para a remoção de sólidos grosseiros; (ii) não opera com os tanques de aeração $\left(15\right.$ e $\left.25 \mathrm{~m}^{3}\right)$ alternadamente, dado que possui um tanque pulmão $\left(50 \mathrm{~m}^{3}\right)$ para o recebimento do esgoto enquanto o processo de depuração biológica ocorre. A ETE opera com tempo de detenção hidráulica de $02 \mathrm{~h} 05$ (intervalo de tempo medido entre a finalização do enchimento e início do descarte) e ciclo total de aeração de $01 \mathrm{~h} 20$ divididos em baterias de aeração intermitentes (50\% do tempo aerando a cada 5 minutos). Na operação regular da ETE não são controlados os parâmetros oxigênio dissolvido (OD), idade do lodo e série de sólidos, porém, mesmo com estas deficiências, a ETE apresenta a eficiência necessária para atendimento das legislações aplicáveis.

\subsection{Caracterização da ETE e do esgoto}

Informações sobre o desempenho da ETE na remoção de matéria orgânica, nitrogênio e fósforo e carga de alimentação foram obtidas pela realização de análises químicas utilizando os procedimentos descritos em APHA et al. (1998) para a determinação de DBO, DQO, nitrogênio total Kjeldahl (NTK) e fósforo total.

\subsection{Amostragem}

Foram realizadas sete campanhas de amostragem entre junho de 2014 e outubro de 2015 em dias de semana e em horário comercial (entre 08 e 18 horas). A cada campanha de 
amostragem, foram coletados 3 litros de esgoto na entrada do tanque de aeração e 3 litros de efluente na linha de descarte.

As coletas foram pontuais, visto a retenção anterior ao tratamento pelo tanque pulmão e a operação do tratamento em ciclos (batelada) que equaliza os conteúdos.

\subsection{Determinação dos hormônios}

\subsubsection{Reagentes e insumos}

Foram utilizados cartuchos de extração em fase sólida (EFS) C18 (500 mg e $3 \mathrm{~mL}$ ), metanol e acetonitrila grau HPLC, água ultra-pura produzida em sistema de purificação Milli-Q, estrona (E1, 99\% pureza), 17- $\beta$-estradiol (E2, 98\% pureza), $17-\alpha$-etinilestradiol (EE2, $98 \%$ pureza) e estriol (E3, 98\% pureza) e filtros em fibra de vidro de 1,2 $\mu \mathrm{m}, 0,7 \mu \mathrm{m}$ e $0,45 \mu \mathrm{m}$.

Foram preparadas soluções estoque, $1000 \mathrm{mg} \mathrm{L}^{-1}$, de cada um dos padrões pela pesagem dos padrões e adição de acetonitrila em balões de 25,00 mL. Estas soluções foram armazenadas sob refrigeração e ao abrigo da luz.

Soluções de trabalho, $10 \mathrm{mg} \mathrm{L}^{-1}$, contendo os 4 hormônios (E1, E2, E3 e EE2) foram preparadas a partir das soluções estoque no dia de uso utilizando micropipetador, balão volumétrico de $10,00 \mathrm{~mL}$ e acetonitrila.

\subsubsection{Preparo das amostras}

As amostras de esgoto $(750 \mathrm{~mL})$ e de efluente $(1000 \mathrm{~mL})$ foram filtradas a vácuo em filtros de fibra de vidro de $1,2 \mu \mathrm{m}$ e depois de $0,7 \mu \mathrm{m}$ e armazenadas sob refrigeração por no máximo 12 horas. $\mathrm{O}$ pH foi corrigido para 3,0 com solução de $\mathrm{HCl}$ 0,1 M após atingir a temperatura ambiente.

Os cartuchos $\mathrm{C} 18$ foram colocados no manifold para EFS e condicionados pela aplicação de 7,00 mL de acetonitrila, 5,00 mL de metanol e 5,00 $\mathrm{mL}$ de água ultra-pura sob vácuo.

As amostras foram aplicadas nos cartuchos utilizando vácuo. Após a passagem de todo o volume de amostras, parte das impurezas foram removidas com a adição de 10,00 mL de solução 1:10 de metanol em água ultra-pura sem vácuo, os cartuchos foram secos por 10 minutos à vácuo e estocados sob refrigeração e ao abrigo da luz.

Utilizando o manifold de EFS, os cartuchos foram eluídos com 4,00 mL de acetonitrila e o eluato foi recolhido em balões de $5,00 \mathrm{~mL}$. A eluição foi realizada após os cartuchos terem sido aclimatados a temperatura ambiente.

O eluato foi avolumado com acetonitrila para $5,00 \mathrm{~mL}$ em balão volumétrico e fracionado em alíquotas de 0,850 mL com o uso de micropipetador em 5 balões de 1,00 mL. A cada um dos balões foi adicionado uma quantidade predeterminada da solução $10 \mathrm{mg} \mathrm{L}^{-1}$ dos hormônios e avolumado para $1,00 \mathrm{~mL}$ com acetonitrila. $\mathrm{O}$ método de adição de padrão ao eluato foi necessário para minimizar o efeito matriz.

\subsubsection{Análise Cromatográfica}

As análises cromatográficas foram realizadas em Cromatógrafo Profissional IC 850 Metrohm com detector Profissional UV-VIS 887, utilizando coluna C18 Prontosil 120-5-C18-AQ-150/4.0 $\mathrm{mm}$ a $30^{\circ} \mathrm{C}$ e fase móvel isocrática $50 \%$ água ultra-pura e $50 \%$ acetonitrila em fluxo de $1 \mathrm{ml} \mathrm{min}{ }^{-1}$. O volume de injeção foi $90 \mu \mathrm{L}$ e a detecção foi a $230 \mathrm{~nm}$.

O uso de cromatografia líquida acoplada a detector UV na separação e quantificação dos hormônios foi à alternativa adotada que possibilitou menores custos operacional e de implantação e a realização deste trabalho. Para contornar o problema de menor detecção deste tipo de equipamento, quando comparado a outros tipos de detectores, como os detectores por 
fluorescência ou por espectrometria de massa, foram utilizados volumes maiores de amostras ( $750 \mathrm{~mL}$ de esgoto e $1000 \mathrm{~mL}$ de efluente).

Os métodos de preparo da amostra e cromatográfico foram validados previamente por Teixeira (2016).

\section{RESULTADOS E DISCUSSÃO}

\subsection{Caracterização do esgoto}

Ao longo das sete coletas realizadas foi verificado que, em média, o esgoto da EEL/USP se apresentou menos concentrado em matéria orgânica (DQO e DBO5) e fósforo total que a média de 166 esgotos recebidos em ETEs municipais instaladas nos estados de Minas Gerais e São Paulo consolidados por Oliveira e Von Sperling (2011). Por outro lado, a concentração de nitrogênio total Kjeldahl (NTK) é superior ao relatado para esgotos sanitários. Os resultados médios destes parâmetros podem ser verificados na Tabela 1.

Os resultados analíticos encontrados (menor concentração de DQO, DBO5 e fósforo e elevada concentração de nitrogênio), podem ser consequência do perfil do campus (sem moradia e preparo de refeições) e podem sugerir uma maior participação da urina neste esgoto em relação aos esgotos municipais avaliados por Oliveira e Von Sperling (2011) e podem interferir na quantidade dos hormônios estrogênicos visto que estes são descartados principalmente na urina (D'Ascenzo et al., 2003).

Tabela 1. Comparativo entre o esgoto da EEL/USP e os resultados médios para alguns parâmetros de caracterização do esgoto recebidos em ETEs municipais.

\begin{tabular}{lcc}
\hline Parâmetros & Esgoto EEL/USP & *Média de 166 ETEs \\
\hline DBO5 $\left(\mathrm{mg} \mathrm{L}^{-1}\right)$ & $123 \pm 5$ & $339 \pm 135$ \\
DQO $\left(\mathrm{mg} \mathrm{L}^{-1}\right)$ & $194 \pm 92$ & $639 \pm 326$ \\
Fósforo $\left(\mathrm{mg} \mathrm{L}^{-1}\right)$ & $3,1 \pm 1,9$ & $3,9 \pm 3,0$ \\
NTK $\left(\mathrm{mg} \mathrm{L}^{-1}\right)$ & $55 \pm 30$ & $50 \pm 17$ \\
\hline
\end{tabular}

*Fonte: Oliveira e Von Sperling (2011).

\subsection{Caracterização da ETE EEL/USP}

A ETE apresentou bom desempenho na remoção de DQO, entre 65 e 95\%, e desempenhos irregulares para a remoção dos nutrientes: entre -54 e $61 \%$ de NTK e entre - 18 e $99 \%$ de fósforo total.

As eficiências médias de remoção de DQO e fósforo, obtidas nesse trabalho, estão em faixas similares às relatadas (81 e 46\%, respectivamente) por Oliveira e Von Sperling (2011), quando avaliado o desempenho de 13 ETEs com tratamento por lodos ativados, em operação no sudeste brasileiro. Ao passo que, quando avaliado a remoção de nitrogênio total Kjeldahl (NTK), a média de remoção relatada por Oliveira e Von Sperling (2001) (entre 54 a 61\%) é superior ao do corrente trabalho. Esses autores, porém não detalham a operação das ETEs avaliadas para que possam ser comparadas ao desempenho da ETE deste trabalho para a remoção de NTK.

A remoção de nitrogênio pode ser melhorada, com impacto positivo na remoção de hormônios, com melhor controle nas etapas sequencias (anóxico/aeróbio) e na idade de lodo (Carballa et al., 2004). Porém, não há na ETE estudada controles que verifiquem o atendimento a estas condições, medidores de potencial de oxi-redução (POR) e de oxigênio dissolvido (OD), podendo ser a causa para o desempenho insatisfatório. 


\subsection{Presença dos hormônios estrogênicos livres no esgoto bruto}

As concentrações médias e as frequências de determinação de cada um dos hormônios são apresentadas na Tabela 2, onde se constata que neste estudo os hormônios E1, E2, E3 e EE2 foram determinados em concentrações superiores aos relatados na literatura consultada, usualmente na faixa de ng L ${ }^{-1}$ (Carballa et al., 2004; Janex-Habibi et al., 2009; Fonseca et al., 2013).

As concentrações de hormônios estrogênicos também foram superiores aos relatados por Stanford e Weinberg (2010) em 5 diferentes ETEs descentralizadas dos EUA, na faixa de ng $\mathrm{L}^{-1}$. O esgoto das ETEs avaliadas por estes autores atendiam também escolas, universidades e escritórios, todos com ocupação em parte do dia.

Tabela 2. Concentrações médias em $\mu \mathrm{g} \mathrm{L}^{-1}$, desvio padrão e frequência de determinação dos hormônios em esgoto bruto.

\begin{tabular}{lcccc}
\hline & E1 & E2 & E3 & EE2 \\
\hline Média $\left(\mu \mathrm{g} \mathrm{L}^{-1)}\right.$ & 5,148 & 7,434 & 5,200 & 5,688 \\
Desvio padrão & 2,747 & 4,356 & 3,331 & 4,312 \\
Frequência & $100 \%$ & $100 \%$ & $75 \%$ & $100 \%$ \\
\hline
\end{tabular}

As concentrações dos hormônios em esgoto determinadas por Ruchiraset e Chinwetkitvanich (2014), em estudo realizado na Tailândia, também foram determinadas na faixa de $\mu \mathrm{g} \mathrm{L}^{-1}$, porém com uma menor frequência de detecção. Os picos de concentrações foram associados a períodos climáticos secos, visto que as amostras sem detecção dos hormônios foram coletadas em períodos chuvosos, o que mostraria o efeito do aumento da precipitação pluviométrica como fator de diluição de esgotos sanitários e dos hormônios.

No Brasil, também foram encontradas maiores concentrações dos hormônios em regiões de seca, o que pode ser exemplificado pelo trabalho de Pessoa et al. (2014), realizado no Ceará, região seca, que encontrou concentrações máximas dos hormônios estrogênicos entre $0,776 \mu \mathrm{g} \mathrm{L}^{-1}$ de E2 a 3,180 $\mu \mathrm{g} \mathrm{L}^{-1}$ de EE2. Já as concentrações encontradas de E1 e E2 por Ferreira (2013) em esgoto do Rio de Janeiro estiveram na faixa de $n g \mathrm{~L}^{-1}$. No corrente trabalho, além das amostras terem sido coletadas em período climático de seca, também foram provenientes de um campus universitário com reduzido consumo de água e geração de esgoto (estimado em $47 \mathrm{~L}$ pessoa $^{-1}$ dia $^{-1}$ ). Estas considerações reforçam o impacto da diluição, por chuva e outros consumos de água, na concentração destes compostos.

Concentrações ainda maiores foram relatadas por Kvanli et al. (2008) para os hormônios em esgoto: até $36,1 \mu \mathrm{g} \mathrm{L}{ }^{-1}$ de E1, até 40,3 $\mu \mathrm{g} \mathrm{L} \mathrm{L}^{-1}$ de E2 e abaixo de 20,0 $\mu_{\mathrm{g} \mathrm{L}}^{-1}$ de E3 e de EE2. Este estudo utilizou urina coletada de grupos de jovens para compor duas amostras de esgoto a ser aplicado em duas diferentes estações de reciclagem de água em escala de laboratório, similares às utilizadas em ônibus espaciais. Os autores acreditam que a utilização apenas de urina, sem outras fontes de água para diluição e a faixa etária dos doadores foram os motivos para as elevadas concentrações. Isto porque os estrogênios são descartados majoritariamente pela urina.

Neste trabalho, o elevado teor de NTK nas amostras de esgoto em relação a outros esgotos municipais, a baixa geração de esgoto per capita e a utilização diferenciada de água no Campus podem indicar um peso maior da urina no esgoto gerado, de forma semelhante ao estudo de Kvanli et al. (2008), e deve ser a causa principal da elevada concentração dos hormônios estrogênicos determinada. 


\subsection{Presença dos hormônios estrogênicos livres no efluente}

Os hormônios estrogênicos foram detectados em concentrações tão elevadas no efluente quanto as determinadas no esgoto e nas mesmas frequências de detecção, como pode ser verificado na Tabela 3. A desconjugação às formas livres, a perda dos grupamentos sulfato ou glicuronídeos com os quais os hormônios estão ligados a serem excretados durante o tratamento biológico e a remoção insuficiente pelo tratamento são as possíveis causas destes altos valores.

$\mathrm{O}$ percurso da rede de esgoto, entre os sanitários e a entrada do tratamento biológico no campus da EEL/USP, pode ter sido insuficiente para que os hormônios fossem desconjugados às formas livres. Isso porqueos glucorohormônios excretados na urina são desconjugados às formas livres dos hormônios ao entrar em contato com os micro-organismos do trato intestinal, principalmente a E. coli, proveniente das fezes. Por outro lado, os sulfatohormônios, outra forma dos hormônios excretados na urina, são mais persistentes. O que pode ser verificado pelo trabalho de D'Ascenzo et al. (2003), no qual foram necessárias 60 horas para a redução dos sulfatohormônios a 50\% da concentração inicial quando em contato com o esgoto e 5 horas para atingir a mesma redução dos glucorohormônios. Durante o tratamento biológico, os sulfatohormônios sofreram desconjugação sendo reduzidos a $26 \%$ da concentração inicial e os glucorohormônios foram reduzidos a $2 \%$. Ambas as desconjugações, de glucorohormônios e de sulfatohormônios, ocorrendo durante o tratamento podem ter produzido hormônios livres encobrindo parte do que foi removido, visto que as formas conjugadas não foram quantificadas neste trabalho. Fenômeno semelhante, aumento ou pequena redução dos hormônios livres, foi identificado no trabalho de Kvanli et al. (2008), no qual os autores associaram a baixa remoção dos hormônios ao curto tempo entre a coleta da urina para composição do esgoto e a aplicação nos tratamentos estudados.

Tabela 3. Concentrações médias em $\mu \mathrm{g} \mathrm{L} \mathrm{L}^{-1}$, desvios-padrão e frequências de detecção por hormônio no efluente.

\begin{tabular}{lcccc}
\hline & E1 & E2 & E3 & EE2 \\
\hline Média & 5,062 & 4,191 & 7,743 & 2,550 \\
Desvio padrão & 3,365 & 3,526 & 3,951 & 2,162 \\
Frequência & $100 \%$ & $100 \%$ & $75 \%$ & $100 \%$ \\
\hline
\end{tabular}

Além da desconjugação durante a etapa biológica, a remoção dos hormônios pode ter sido insuficiente, haja visto que as condições relacionadas à eficiente biodegradação destes compostos em tratamento por lodos ativados (idade do lodo) não foram aferidas ou não foram alcançadas (tempo de detenção hidráulica maior e remoção de nitrogênio) neste trabalho. Apenas a baixa carga de alimentação foi constatada dentre os fatores que promoveriam a melhor biodegradação (Johnson et al., 2005; Li et al., 2008; Pessoa et al., 2014).

No estudo de Servos et al. (2005) com 18 ETEs em operação no Canadá, duas destas, operando com sistema de lodos ativados convencional apresentaram condições semelhantes entre si de TDH (aproximadamente 7 horas) e remoção de E2 distintas (40 e 76\%). A ETE de melhor remoção de E2, apesar de não operar com elevada idade de lodo, obteve melhor remoção parcial de nitrogênio ( $85 \%$ contra $45 \%$ da ETE que não obteve bom desempenho).

Uma das 17 ETEs utilizadas no estudo de Johnson et al. (2005), Eindhoven (Ei), possuía sistema de tratamento de lodos ativados com TDH menor do que deste trabalho (1,3 horas). Porém, apresentou remoção de E1 de 94\%, o que pode ser atribuído à existência de zona anóxica e à elevada idade de lodo (25 dias) condições necessária para a desnitrificação. Boas remoções, com médias acima de $85 \%$ de E1 e de E2, também foram determinadas por Janex-Habibi et al. 
(2009) em 9 ETEs por lodos ativados operando na Europa e Estados Unidos. Além do maior TDH, entre 5 e 30 horas, estas possuíam etapas para remoção biológica de nitrogênio.

A baixa carga orgânica alimentada, similar ou inferior aos valores citados nos estudos de Carballa et al. (2004) e Ferreira (2013), pode ter sido o fator determinante para a remoção parcial dos hormônios, E2 e EE2, mesmo em condições adversas de TDH, de desnitrificação e sem acompanhamento da idade de lodo. Fato que é corroborado pelo trabalho de Li et al. (2008) que determinaram taxas de biodegradação dos hormônios E1, E2 e EE2 inversamente proporcionais à concentração de glicose (usada como matéria orgânica) no meio reativo.

Em relação ao hormônio E1, além da desconjugação e da baixa capacidade do tratamento biológico para a remoção dos hormônios estudados, a conversão por oxidação microbiana de outros hormônios estrogênicos a E1 pode ser fundamento adicional para as altas concentrações detectadas no efluente. Carballa et al. (2004) e Servos et al. (2005) também relataram menor remoção do E1 em relação aos outros hormônios estrogênico ou até o surgimento destes em meios nos quais não foram inoculados.

\section{CONCLUSÕES}

O esgoto gerado na EEL-USP e o efluente tratado na estação por lodos ativados em bateladas sequenciais apresentaram altas concentrações dos hormônios estrogênios $\left(5,158 \pm 2,747 ; 7,434 \pm 4,356 ; 5,200 \pm 3,331\right.$ e $5,638 \pm 4,312 \mu \mathrm{g} \mathrm{L}^{-1}$ de E1, E2, E3 e EE2 no esgoto bruto e $5,062 \pm 3,366 ; 4,191 \pm 3,527 ; 7,743 \pm 3,951,2,550 \pm 2,162$ de E1, E2, E3 e EE2 no efluente) e com frequência de detecção próxima a 100\%. Ambos os parâmetros diferem da maioria dos resultados publicados em trabalhos semelhantes que apresentaram concentrações na faixa de $n g L^{-1}$.

As características químicas do esgoto avaliado (elevada concentração de NTK e menores concentrações de matéria orgânica, DBO5 e DQO, e de fósforo em comparação ao esgoto doméstico) e as particularidades do Campus (sem preparo de refeições, sem moradias e com geração per capita de esgoto reduzida, $47 \mathrm{~L} \mathrm{pessoa}^{-1}$ dia $^{-1}$ ) associadas à condição climática nos períodos de coleta (clima seco) podem indicar uma maior participação da urina no esgoto e, consequentemente, justificar as elevadas concentrações dos hormônios estrogênicos determinadas neste trabalho, visto que os hormônios estrogênicos são excretados principalmente pela urina.

As concentrações dos hormônios estrogênicos no efluente, após o tratamento biológico, também foram superiores às encontradas na literatura pesquisada. Isto pode ter acontecido devido às elevadas concentrações de entrada (no esgoto bruto), mas também podem estar associadas à baixa eficiência de remoção dos hormônios. Em sistemas de lodos ativados, as melhores eficiências de remoção dos hormônios estrogênicos têm sido relacionadas ao prolongado TDH, elevada idade do lodo, baixa carga orgânica e caráter desnitrificante do tratamento. Porém, apenas a baixa carga orgânica foi atingida neste trabalho, a remoção de nitrogênio foi insatisfatória e inconstante e a idade do lodo não pode ser medida.

Ademais, os hormônios estrogênicos são excretados pelo corpo humano conjugados a grupamentos sulfatos e glicuronídeos. Grande parte dos grupamentos glicuronídeos pode ser removida pela ação das bactérias do trato intestinal presentes no esgoto demandando tempo de contato e de reação, já os grupamentos sulfatos e o glicuronídeos restante podem ser removidos pelos micro-organismos do lodo ativado durante o tratamento. Visto que a ETE estudada é interna ao Campus, o esgoto é tratado próximo aos pontos de geração, assim o tempo de trânsito pode não ter sido suficiente para a remoção dos grupamentos conjugados antes do tratamento. Desta maneira, os hormônios conjugados não foram quantificados no esgoto bruto, mas passaram a ser quantificados no efluente se a desconjugação ocorreu durante o tratamento por 
lodos ativados, mascarando a remoção dos hormônios livres, que foram os quantificados neste trabalho.

Recomenda-se que novos estudos sejam realizados no qual se determine também as concentrações dos hormônios estrogênicos conjugados para que possa ser aferido o impacto da desconjugação durante o tratamento.

Ademais, a redução nas concentrações dos hormônios no efluente pode ser melhorada com implantação de condições necessárias para a remoção biológica de nitrogênio, como a adoção de acompanhamento da idade de lodo e o controle da aeração por ORP.

Deve ser ressaltado que a ETE em estudo, da mesma maneira que outras ETEs com processo de tratamento convencionais, foi projetada e posteriormente adaptada para reduzir a carga de poluentes orgânicos e não visava especificamente a remoção de contaminantes emergentes presentes no esgoto sanitário, como os hormônios estrogênicos. Assim, qualquer remoção que possa ocorrer desses compostos é eventual e não específica ao processo de tratamento convencional. Os dados determinados nesse estudo mostram a necessidade de se rever o modelo de tratamento de esgoto em uso no campus, com vistas de se estabelecer um novo paradigma para lançamento de seus efluentes na bacia do Rio Paraíba do Sul.

\section{REFERÊNCIAS}

AMERICAN PUBLIC HEALTH ASSOCIATION - APHA; AMERICAN WATER WORKS ASSOCIATION - AWWA; WATER ENVIRONMENT FEDERATION - WEF. Standard methods for the examination of water and wastewater. 20th ed. Washington, 1998.

CARBALLA, M.; OMIL, F.; LEMA, J.; LLOMPART, M.; GARCÍA-JARES, C.; RODRÍGUEZ, I. et al. Behavior of pharmaceuticals, cosmetics and hormones in a sewage $\begin{array}{llllll}\text { treatment plant. Water Research, v. 38, p. 2918-2926, } 2004 . & \end{array}$ https://doi.org/10.1016/j.watres.2004.03.029

D'ASCENZO, G.; DI CORCIA, A.; GENTILI, A.; MANCINI, R.; MASTROPASQUA, R.; NAZZARI, M.; SAMPERI., R. Fate of natural estrogen conjugates in municipal sewage transport and treatment facilities. The Science of the Total Environment, v. 302, p. 199209, 2003. https://doi.org/10.1016/S0048-9697(02)00342-X

FERREIRA, A. Endocrine disruptors in sludge wastewater treatment plants: environmental complications. Acta Scientiarum - Technology, v. 35, n. 2, p. 307-316, 2013. http://dx.doi.org/10.4025/actascitechnol.v35i2.10619

FONSECA, A.; CARDOSO, M.; ESTEVES, V. Determination of Estrogens in Raw and Treated Wastewater by High-Performance Liquid Chromatography- Ultraviolet Detection. Journal of Environmental e Analytical Toxicology. v. 4, n. 1, p. 1-5, 2013.

JANEX-HABIBI, M. L.; HUYARD, A.; ESPERANZA, M.; BRUCHET, A. Reduction of endocrine disruptor emissions in the environment. Water Research, v. 43, p. 1565-1576, 2009. https://doi.org/10.1016/j.watres.2008.12.051

JOHNSON, A. C.; AERNI, H. R.; GERRITSEN, A.; GIBERT, M.; GIGER, W.; HYLLAND, $\mathrm{K}$. et al. Comparing steroid estrogen, and nonylphenol content across a range of European sewage plants with different treatment and management practices. Water Research, v. 39, p.47-58, 2005. https://doi.org/10.1016/j.watres.2004.07.025 
KVANLI, D. M.; MARISETTY, S.; ANDERSON, T. A.; JACKSON, W. A.; MORSE, A. N. Monitoring Estrogen Compounds in Wastewater Recycling Systems. Water Air Soil Pollution, v. 188, p. 31-40, 2008. https://doi.org/10.1007/s11270-007-9498-6

LI, F.; DESMIARTI, R.; YUASA, A.; HORIO, A. Behavior of natural estrogens in semicontinuous activated sludge biodegradation reactors. Bioresource Technology, v. 99, p. 2964-2971, 2008. https://doi.org/10.1016/j.biortech.2007.06.016

OLIVEIRA, S.; VON SPERLING, M. Performance evaluation of different wastewater treatment technologies operating in a developing country. Journal of Water, Sanitation and Hygiene for Development, v. 01, n. 01, p. 37-56, 2011. http://dx.doi.org/10.2166/washdev.2011.022

PESSOA, G. P.; SOUZA, N. C.; VIDAL, C. B.; ALVES, J. A. C.; FIRMINO, P. I. M.; NASCIMENTO, R. F. et al. Occurrence and removal of estrogens in Brazilian wastewater treatment plants. The Science of the total environment, v. 490, p. 288-295, 2014. https://doi.org/10.1016/j.scitotenv.2014.05.008

RUCHIRASET, A.; CHINWETKITVANICH, S. Occurrence of estrogens in wastewater treatment tlants and surface water in Bangkok area, Thailand. Advanced Materials $\begin{array}{lllll}\text { Research, } & \text { v. } & 931-932, & \text { p. } & 721-726,\end{array}$ http://dx.doi.org/10.4028/www.scientific.net/AMR.931-932.721

SERVOS, M. R.; BENNIE, D. T.; BURNISON, B. K.; JURKOVIC, A.; McINNIS, R.; NEHELI, T. et al. Distribution of estrogens, 17h-estradiol and estrone, in Canadian municipal wastewater treatment plants. Science of the Total Environment, v. 336, p. 155-170, 2005. https://doi.org/10.1016/j.scitotenv.2004.05.025

STANFORD, B. D.; WEINBERG, H. S. Evaluation of OnSite Wastewater Treatment Technology to Remove Estrogens, Nonylphenols, and Estrogenic Activity from Wastewater. Environmental Science \& Technology, v. 44, n. 8, p. 2994-3001, 2010. http://dx.doi.org/10.1021/es903422b

TEIXEIRA, R. B. Estudo da presença e remoção de hormônios estrogênicos em estação de tratamento de esgoto por lodos ativados. 2016. 85 p. Dissertação (Mestrado em Ciências) - Escola de Engenharia de Lorena, Universidade de São Paulo, Lorena, 2016. 